\title{
FaceHop: A Light-Weight Low-Resolution Face Gender Classification Method
}

\author{
Mozhdeh Rouhsedaghat ${ }^{1}$, Yifan Wang ${ }^{1}$, Xiou Ge ${ }^{1}$, Shuowen $\mathrm{Hu}^{2}$, Suya You ${ }^{2}$, \\ and C.-C. Jay Kuo ${ }^{1}$ \\ 1 University of Southern California, Los Angeles, California, USA \\ \{rouhseda, wang608, xiouge, jckuo\}@usc.edu \\ 2 Army Research Laboratory, Adelphi, Maryland, USA \\ \{shuowen.hu.civ, suya.you.civ\}@mail.mil
}

\begin{abstract}
A light-weight low-resolution face gender classification method, called FaceHop, is proposed in this research. We have witnessed rapid progress in face gender classification accuracy due to the adoption of deep learning (DL) technology. Yet, DL-based systems are not suitable for resource-constrained environments with limited networking and computing. FaceHop offers an interpretable non-parametric machine learning solution. It has desired characteristics such as a small model size, a small training data amount, low training complexity, and low-resolution input images. FaceHop is developed with the successive subspace learning (SSL) principle and built upon the foundation of PixelHop++. The effectiveness of the FaceHop method is demonstrated by experiments. For gray-scale face images of resolution $32 \times 32$ in the LFW and the CMU Multi-PIE datasets, FaceHop achieves correct gender classification rates of $94.63 \%$ and $95.12 \%$ with model sizes of $16.9 \mathrm{~K}$ and $17.6 \mathrm{~K}$ parameters, respectively. It outperforms LeNet- 5 in classification accuracy while LeNet-5 has a model size of $75.8 \mathrm{~K}$ parameters.
\end{abstract}

Keywords: gender classification - light-weight model- small data. SSL PixelHop++.

\section{Introduction}

Face attributes classification is an important topic in biometrics. The ancillary information of faces such as gender, age, and ethnicity is referred to as soft biometrics in forensics [1213. The face gender classification problem has been extensively studied for more than two decades. Before the resurgence of deep neural networks (DNNs) around 7-8 years ago, the problem was treated using the standard pattern recognition paradigm. It consists of two cascaded modules: 1) unsupervised feature extraction and 2) supervised classification via common machine learning tools such as support vector machine (SVM) and random forest (RF) classifiers.

We have seen fast progress on this topic due to the application of deep learning (DL) technology in recent years. Generally speaking, cloud-based face verification, recognition, and attributes classification technologies have become mature, and they have been used in many real world biometric systems. Convolution 
neural networks (CNNs) offer high-performance accuracy. Yet, they rely on large learning models consisting of several hundreds of thousands or even millions of model parameters. The superior performance is contributed by factors such as higher input image resolutions, more and more training images, and abundant computational/memory resources.

Edge/mobile computing in a resource-constrained environment cannot meet the above-mentioned conditions. The technology of our interest finds applications in rescue missions and/or field operational settings in remote locations. The accompanying face inference tasks are expected to execute inside a poor computing and communication infrastructure. It is essential to have a smaller learning model size, lower training and inference complexity, and lower input image resolution. The last requirement arises from the need to image individuals at farther standoff distances, which results in faces with fewer pixels.

In this work, we propose a new interpretable non-parametric machine learning solution called the FaceHop method. FaceHop has quite a few desired characteristics, including a small model size, a small training data amount, low training complexity, and low-resolution input images. FaceHop follows the traditional pattern recognition paradigm that decouples the feature extraction module from the decision module. However, FaceHop automatically extracts statistical features instead of handcrafted features. It is developed with the successive subspace learning (SSL) principle 4 45]6 and built upon the foundation of the Pixel-

Hop++ system [7. The effectiveness of the FaceHop method is demonstrated by experiments on two benchmarking datasets. For gray-scale face images of resolution $32 \times 32$ obtained from the LFW and the CMU Multi-PIE datasets, FaceHop achieves gender classification accuracy of $94.63 \%$ and $95.12 \%$ with model sizes of $16.9 \mathrm{~K}$ and $17.6 \mathrm{~K}$ parameters, respectively. FaceHop outperforms LeNet-5 while the LeNet- 5 model is significantly larger and contains $75.8 \mathrm{~K}$ parameters.

There are three main contributions of this work. First, it offers a practical solution to the challenging face biometrics problem in a resource-constrained environment. Second, it is the first effort that applies SSL to face gender classification and demonstrates its superior performance. Third, FaceHop is fully interpretable, non-parametric, and non-DL-based. It offers a brand new path for research and development in biometrics.

The rest of this paper is organized as follows. Related work is reviewed in Sec. 2. The FaceHop method is presented in Sec. 3 Experimental set-up and results are detailed in Sec. 4. Finally, concluding remarks and future extensions are given in Sec. 5 .

\section{Related Work}

\subsection{Face Attributes Classification}

We can classify face attributes classification research into two categories: nonDL-based and DL-based. DL-based solutions construct an end-to-end parametric model (i.e. a network), define a cost function, and train the network to minimize 
the cost function with labeled face gender images. The contribution typically arises from a novel network design. Non-DL-based solutions follow the pattern recognition paradigm and their contributions lie in using different classifiers or extracting new features for better performance.

Non-DL-based Solutions. Researchers have studied different classifiers for gender classification. Gutta et al. 8] proposed a face-based gender and ethnic classification method using the ensemble of Radial Basis Functions (RBF) and Decision Trees (DT). SVM [9] and AdaBoost [10, have been studied for face gender classification. Different feature extraction techniques were experimented to improve classification accuracy. A Gabor-kernel partial-least squares discrimination (GKPLSD) method for more effective feature extraction was proposed by Štruc et al. [11. Other handcrafted features were developed for face gender classification based on the local directional patterns (LDP) [12] and shape from shading [13. Cao et al. [14] combined Multi-order Local Binary Patterns (MOLBP) with Localized Multi-Boost Learning (LMBL) for gender classification.

Recent research has focused more on large-scale face image datasets. Li et al. [15] proposed a novel binary code learning method for large-scale face image retrieval and facial attribute prediction. Jia et al. [16] collected a large dataset of 4 million weakly labeled face in the wild (4MWLFW). They trained the CPegasos classifier with Multiscale Local Binary Pattern (LBP) features using the 4MWLFW dataset and achieved the highest test accuracy on the LFW dataset for Non-DL-based methods up to now. Fusion of different feature descriptors and region of interests (ROI) were examined by Castrillón-Santana et al. [17].

The mentioned methods either have a weak performance as a result of failing to extract strong features from face images or have a large model size.

DL-based Solutions. With the rapid advancement of the DL technology, DL-based methods become increasingly popular and achieve unprecedented accuracy in face biometrics [18. Levi et al. [19] proposed a model to estimate age and gender using a small training data. Duanet al. 20] introduced a hybrid CNN-ELM structure for age and gender classification which uses CNN for feature extraction from face images and ELM for classifying the features. Taherkhani et al. 21] proposed a deep framework which predicts facial attributes and leveraged it as a soft modality to improve face identification performance. Han et al. 22] investigated the heterogeneous face attribute estimation problem with a deep multi-task learning approach. Ranjan et al. 23] proposed a multi-task learning framework for joint face detection, landmark localization, pose estimation, and gender recognition. Antipov et al. 24] investigated the relative importance of various regions of human faces for gender and age classification by blurring different parts of the faces and observing the loss in performance. ResNet50 [24, AlexNet [25, and VGG16 [18] were applied to gender classification of the LFW dataset, and decent performance was observed. However, these models have very large model sizes. Considerable amounts of computation and storage resources are required to implement these solutions. 
Light-Weight CNNs. Light-weight networks are significantly smaller in size than regular networks while achieving comparable performance. They find applications in mobile/edge computing. One recent development is the SqueezeNet [26] which achieves comparable accuracy with the AlexNet [27] but uses 50x fewer parameters. It contains $4.8 \mathrm{M}$ model parameters. In the area of face recognition, Wu et al. 28 proposed a light CNN architecture that learns a compact embedding on a large-scale face dataset with massive noisy labels. Although the mentioned models are relatively small, they still require a large amount of training data.

\subsection{Successive Subspace Learning (SSL)}

Representation learning plays an important role in many representation learning methods are built upon DL, which is a supervised approach. It is also possible to use an unsupervised approach for representation learning automatically (i.e. not handcrafted). For example, there exist correlations between image pixels and their correlations can be removed using the principal component analysis (PCA). The application of PCA to face images was introduced by Turk and Pentland [29]. The method is called the "Eigenface". One main advantage of converting face images from the spatial domain to the spectral domain is that, when face images are well aligned, the dimension of input face images can be reduced significantly and automatically. Since we attempt to find a powerful subspace for face image representation, it is a subspace learning method.

Chan et al. 30 proposed a PCANet that applies the PCA to input images in two stages. Chen et al. 31 proposed a PixelHop system that applies cascaded Saab transforms 6 to input images in three stages, where the Saab transform is a variant of the PCA that adds a positive bias term to avoid the sign confusion problem 44. The main difference between Eigenface, PCANet and PixelHop is to conduct the PCA transform in one, two, or multiple stages. If we apply one-stage PCA, the face is a pure spatial- and spectral-domain representations before and after the transform, respectively. Since the spatial representation is local, it cannot offer the global contour and shape information easily. On the contrary, the spectral representation is global, it fails to differentiate local variations. It is desired to get multiple hybrid spatial/spectral representations. This can be achieved by multi-stage transforms. Kuo et al. developed two multistage transforms, called the Saak transform [32] and the Saab transform [6], respectively. Recently, the channel-wise (c/w) Saab transform was proposed in 7 to enhance the efficiency of the Saab transform.

Inspired by the function of convolutional layers of CNNs [a], the PixelHop system 31] and the PixelHop++ system [7] were developed to serve the same function but derived based on a completely different principle. The weights of convolutional filters in CNNs are obtained by end-to-end optimization through backpropagation. In contrast, the convolutional kernels used in PixelHop and PixelHop ++ are the Saab filters. They are derived by exploiting statistical correlations of neighboring pixels. As a result, both PixelHop and PixelHop++ are 


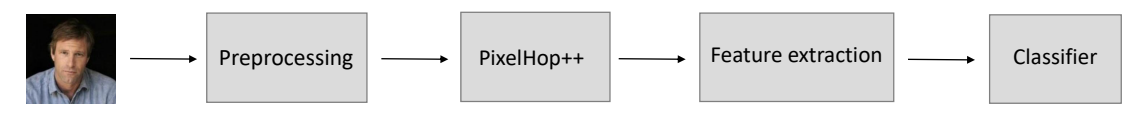

Fig. 1. An overview of the proposed FaceHop method.

fully unsupervised. Neither label nor backpropagation is needed in filter weights computation.

The PixelHop++ system 7 is an enhanced version of the PixelHop system 31. The main difference between PixelHop and PixelHop++ is that the former uses the Saab transform while the latter adopts the c/w Saab transform. The c/w Saab transform requires fewer model parameters than the Saab transform since channels are decoupled in the c/w Saab transform.

\section{Proposed FaceHop Method}

An overview of the proposed FaceHop system is shown in Fig. 1. It consists of four modules: 1) Preprocessing, 2) PixelHop++, 3) Feature extraction, and 4) Classification. Since PixelHop++ is the most unique module in our proposed solution for face gender classification, it is called the FaceHop system. The functionality of each module will be explained below in detail.

\subsection{Preprocessing}

Face images have to be well aligned in the preprocessing module to facilitate their processing in the following pipeline. In this work, we first use the dlib [33] tool for facial landmarks localization. Based on detected landmarks, we apply a proper $2 \mathrm{D}$ rotation to each face image to reduce the effect of pose variation. Then, all face images are centered and cropped to remove the background. Afterwards, we apply histogram equalization to each image to reduce the effect of different illumination conditions. Finally, all images are resized to a low resolution one of $32 \times 32$ pixels.

\subsection{PixelHop ++}

Both PixelHop and PixelHop++ are used to describe local neighborhoods of a pixel efficiently and successively. The size of a neighborhood is characterized by the hop number. One-hop neighborhood is the neighborhood of the smallest size. Its actual size depends on the filter size. For example, if we use a convolutional filter of size $5 \times 5$, then the hop- 1 neighborhood is of size $5 \times 5$. The Saab filter weights are obtained by performing dimension reduction on the neighborhood of 


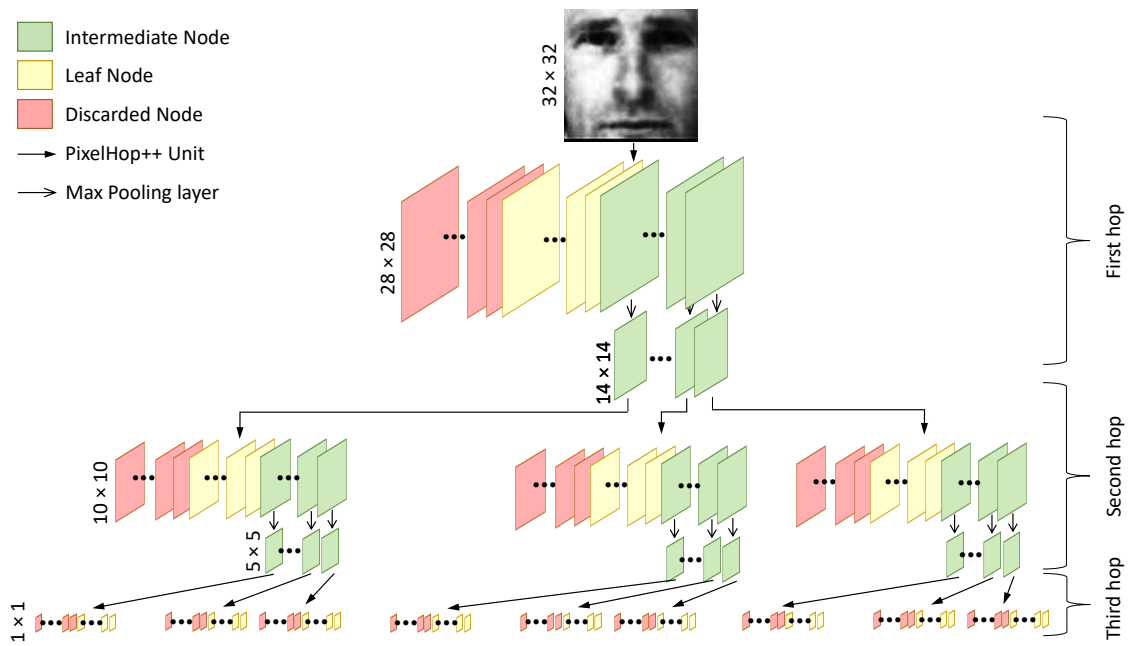

Fig. 2. Illustration of the proposed 3-hop FaceHop system as a tree-decomposed representation with its depth equal to three, where each depth layer corresponds to one hop.

a target pixel using PCA. The Saab filters in PixelHop and PixelHop++ serve as an equivalent role of convolutional filters in CNNs. For example, a neighborhood of size $5 \times 5$ has a dimension of 25 in the spatial domain. We can use the Saab transform to reduce its original dimension to a significantly lower one. We should mention that the neighborhood concept is analogous to the receptive field of a certain layer of CNNs. As we go to deeper layers, the receptive field becomes larger in CNNs. In the SSL context, we say that the neighborhood size becomes larger as the hop number increases.

The proposed 3-hop PixelHop++ system is shown in Fig. 2, which is a slight modification of [7] so as to tailor to our problem. The input is a gray-scale face image of size $32 \times 32$. Each hop consists of a PixelHop++ unit followed by a $(2 \times$ 2)-to- $(1 \times 1)$ max-pooling operation. A PixelHop++ system has three ingredients: 1) successive neighborhood construction, 2) channel-wise Saab transform, and 3) tree-decomposed feature representation. They are elaborated below.

1) Successive neighborhood construction. We need to specify two parameters to build the neighborhood of the center pixel at each hop. There are the window-size and the stride. We use a window size of $5 \times 5$ and stride of 1 in all three hops in Fig. 2. The neighborhood size grows bigger as the hop number becomes larger due to the max-pooling operation. The first, second, and third hops characterize the information of the short-, mid-, and long-range neighborhoods of the center pixel. Apparently, each neighborhood has a degree of freedom of 25 in the spatial domain. By collecting these neighborhood samples from different spatial locations, we can study their statistical correlations via a covariance matrix of dimension $25 \times 25$. Then, we conduct the eigenvector/eigenvalue analysis 
to the covariance matrix to find a more economical representation. That is, we can convert pixel values from the spatial domain to the spectral domain, which leads to the PCA transform, for dimension reduction.

2) Channel-wise (c/w) Saab transform. The PCA transform has both positive and negative responses. We encounter a sign-confusion problem [4] when a convolutional operation in the $(i+1)$ th stage has the sum of two terms: 1) a positive response in the $i$ th stage multiplied by a positive outgoing link and 2) a negative response in the $i$ th stage multiplied by a negative outgoing link. Both terms contribute positive values to the output while their input patterns are out of phase. Similarly, there will be another sign-confusion when the convolutional operation in the $(i+1)$ th stage has the sum of a positive response multiplied by a negative filter weight as well as a negative response multiplied by a positive filter weight. They both contribute to negative values.

To resolve such confusion cases, a constant bias term is added to make all responses positive. This is called the Saab (subspace approximation via adjusted bias) transform [6]. Typically, the input of the next pixelhop unit is a 3D tensor of dimension $N_{x} \times N_{y} \times k$, where $N_{x}=N_{y}=5$ are spatial dimensions of a filter and $k$ is the number of kept spectral components. The Saab transform is used in PixelHop.

Since channel responses can be decorrelated by the eigen analysis, we are able to treat each channel individually. This results in channel-wise (c/w) Saab transform [7. The main difference between the standard Saab and the c/w Saab transforms is that one $3 \mathrm{D}$ tensor of dimension $N_{x} \times N_{y} \times k$ can be decomposed into $k 2 \mathrm{D}$ tensors of dimension $N_{x} \times N_{y}$ in the latter. Furthermore, responses in higher frequency channels are spatially uncorrelated so that they do not have to go to the next hop. The c/w Saab transform is used in PixelHop++. It can reduce the model size significantly as compared with the Saab transform while preserving the same performance.

3) Tree-decomposed representation. Without loss of generality, we use the first hop to explain the c/w Saab transform design. The neighborhood of a center pixel contains 25 pixels. In the spectral domain, we first decompose it into the direct sum of two orthogonal subspaces - the DC (direct current) subspace and the AC (alternating current) subspace. Then, we apply the PCA to the AC subspace to derive Saab filters. After the first-stage Saab transform, we obtain one DC coefficient and $24 \mathrm{AC}$ coefficients in a grid of size $28 \times 28$. We classify AC coefficients into three groups based on their associated eigenvalues: low-, mid-, and high-frequency $\mathrm{AC}$ coefficients.

When the eigenvalues are extremely small, we can discard responses in these channels without affecting the quality of the input face image. This is similar to the eigenface approach in spirit. For mid-frequency AC coefficients, the spatial correlation of their responses is too weak to offer a significant response in hop- 2 . Thus, we can terminate its further transform. For low-frequency AC coefficients, the spatial correlation of their responses is strong enough to offer a significant response in hop- 2 . Then, we conduct max-pooling and construct the hop- 2 neighborhood of these frequency channels in a grid of size $14 \times 14$. It is easy to show 


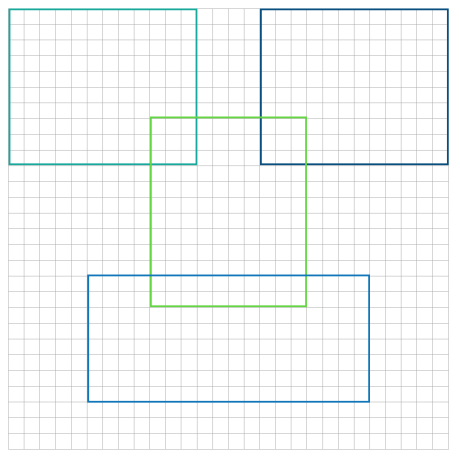

(a)

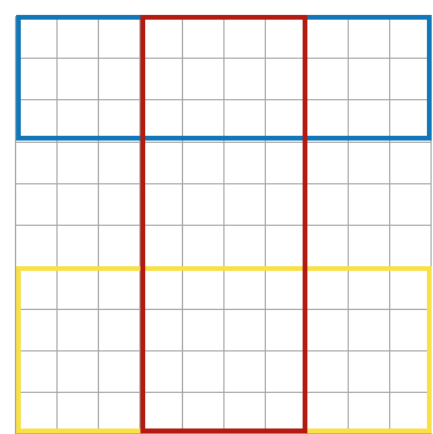

(b)

Fig. 3. Collection of regional responses in hop-1 and hop- 2 response maps as features in the FaceHop system: (a) four regions in hop-1 and (b) three regions in hop- 2 .

these hop-by-hop operations using a tree. Then, each channel corresponds to a node. We use the green, yellow and pink colors to denote low-, mid- and highfrequency AC channels in Fig. 2 where the DC channel is also colored in green. They are called the intermediate, leaf, and discard nodes in a hierarchical tree of depth equal to three.

To determine which node belongs to which group, we use the energy of each node as the criterion. The energy of the root node is normalized to one. The energy of each node in the tree can be computed and normalized against the energy value of the root node. Then, we can choose two thresholds (in terms of energy percentages) at each hop to partition nodes into three types. These energy thresholds are hyperparameters of the PixelHop++ model.

\subsection{Feature Extraction}

Responses at each of the three hops of the FaceHop system have different characteristics. As shown in Fig. 2. Hop-1 has a response map of size $28 \times 28$, Hop-2 has a response map of size $10 \times 10$ and Hop-3 has a response map of size $1 \times 1$. Hop- 1 responses give a spatially detailed representation of the input. Yet, it is difficult for them to offer regional and full views of the entire face unless the dimension of hop- 1 responses becomes extremely large. This is expensive and unnecessary. Hop-2 responses give a coarser view of the entire face so that a small set of them can cover a larger spatial region. Yet, they do not have face details as given by Hop-1 responses. Finally, Hop-3 responses lose all spatial details but provide a single value at each frequency channel that covers the full face. The eigenface approach can only capture responses of the full face and cannot obtain the information offered by hop-1 and hop-2 responses in the FaceHop system. We will extract features based on responses in all three hops. 
We group pixel responses in hop-1 and hop-2 to form region responses as shown in Fig. 3 .

- Hop-1. We collect pixel responses in hop-1 to form four regions as shown in Fig. 3 (a). They cover the left eye, the right eye, the nose, and the mouth regions. Their spatial dimensions (height versus width) are $10 \times 12,10 \times 12$, $12 \times 10$ and $8 \times 18$, respectively. There are spatial correlations for responses of the same channel. Thus, we can apply another PCA to responses of the same hop/region for dimension reduction. Usually, we can reduce the dimension to the range between 15 and 20. Afterwards, we concatenate the reduced dimension vector of each region across all hop-1 channels (including both leaf and intermediate nodes) to create a hop/region feature vector and feed it to a classifier. There are four hop-1 regions, and we have four feature vectors that contain both spatial and spectral information of a face image. The dimension of hop-1 feature vectors in four regions will be given in Table 2 .

- Hop-2. We collect pixel responses in hop-2 to form three regions as shown in Fig. 3 (b). They are: one horizontal stripe of dimension $3 \times 10$ covering two eyes, another horizontal stripe of dimension $4 \times 10$ covering the mouth, and one vertical stripe of dimension $10 \times 4$ covering the nose as well as the central $40 \%$ region. Similarly, we can perform dimension reduction via PCA and concatenate the spatially reduced dimension of each region across all hop-2 channels to train three classifiers. The dimension of hop-2 feature vectors in the three regions will be summarized in Table 2 .

- Hop-3. We use all responses of hop-3 as one feature vector to train a classifier.

It is worthwhile to point out that, although some information of intermediate nodes will be forwarded to the next hop, different hops capture different information contents due to varying spatial resolutions. For this reason, we include responses in both intermediate and leaf nodes at hop- 1 and hop- 2 as features.

\subsection{Classifiers}

As described in Sec. 3.3 we train four classifiers in hop 1, another three classifiers in hop 2, and one classifier in hop 3. Each classifier is a binary classifier. It takes a long feature vector as the input and makes a soft decision, which is the probability for the face to be a male or a female. Since the two probabilities add to unity, we only need to record one of them. Then, at the next stage, we feed these eight probabilities into a meta classifier for the final decision. The choice of classifiers can be the Random Forest (RF), the Support Vector Machine (SVM), and the Logistic Regression (LR). Although the SVM and the RF classifiers often give higher accuracy, they have a larger number of model parameters. Since our interest lies in a smaller model size, we adopt the LR classifier in our experiments only. 


\section{Experiments}

In this section, we evaluate the proposed FaceHop gender classification method. We compare the FaceHop solution with a variant of LeNet-5 in model sizes and verification performance. The reason for choosing the LeNet-5 for performance benchmarking is that it is a small model which is demonstrated to have a relatively high classification accuracy on gray-scale $32 \times 32$ images. The neuron numbers of the modified LeNet-5 model are changed to 16 (1st Conv), 40 (2nd Conv), 140 (1st FC), 60 (2nd FC) and 2 (output). The modification is needed since human faces are more complicated than handwritten digits in the MNIST dataset. For fair comparison, we train both models on the same training data which is achieved by applying the preprocessing and data augmentation to the original face images. We use only logistic regression (LR) classifiers in FaceHop due to its small model size.

Datasets. We adopt the following two face image datasets in our experiments.

- LFW dataset 34

The LFW dataset consists of 13,233 face images of 5,749 individuals, which were collected from the web. There are 1,680 individuals who have two or more images. A 3D aligned version of LFW [35] is used in our experiments.

- CMU Multi-PIE dataset 36.

The CMU Multi-PIE face dataset contains more than 750,000 images of 337 subjects recorded in four sessions. We select a subset of the 01 session that contains frontal and slightly non-frontal face images (camera views $05 \_0$, 05_1, and 14_0) with all the available expressions and illumination conditions in our experiments.

Data Augmentation. Since both datasets have significantly fewer female images, we use two techniques to increase the number of female faces.

- Flipping the face images horizontally.

- Averaging a female face image with its nearest neighbor in the reduced dimension space to generate a new female face image. To find the nearest neighbor, we project all female images to a reduced dimension space, which is obtained by applying PCA and keeping the highest energy components with $90 \%$ of the total energy. Dimension reduction is conducted to eliminate noise and high-frequency components. The quality of augmented female images is checked to ensure that they are visually pleasant.

After augmentation, the number of male images is still slightly more than the number of female images.

Configuration of PixelHop++. The configurations of the PixelHop ++ module for LFW and CMU Multi-PIE datasets are shown in Table 1. We list the numbers of intermediate nodes, leaf nodes and discarded nodes at each hop (see Fig. 2) in the experiments. In our design, we partition channels into two groups (instead of three) only at each hop. That is, they are either discarded or 


\begin{tabular}{|c|l|l|l|l|l|l|}
\hline \multirow{2}{*}{ Hop Index } & \multicolumn{3}{|c|}{ LFW } & \multicolumn{3}{c|}{ CMU Multi-PIE } \\
\cline { 2 - 7 } & $\begin{array}{l}\text { Interm. } \\
\text { Node No. }\end{array}$ & $\begin{array}{l}\text { Leaf } \\
\text { Node No. }\end{array}$ & $\begin{array}{l}\text { Discarded } \\
\text { Node No. }\end{array}$ & $\begin{array}{l}\text { Interm. } \\
\text { Node No. }\end{array}$ & $\begin{array}{l}\text { Leaf } \\
\text { Node No. }\end{array}$ & $\begin{array}{l}\text { Discarded } \\
\text { Node No. }\end{array}$ \\
\hline \hline Hop-1 & 18 & 0 & 7 & 18 & 0 & 7 \\
\hline Hop-2 & 122 & 0 & 328 & 117 & 0 & 333 \\
\hline Hop-3 & 0 & 233 & 2,817 & 0 & 186 & 2,739 \\
\hline
\end{tabular}

Table 1. Configurations of PixelHop++ for LFW and CMU Multi-PIE..

all forwarded to the next hop. As a result, there are no leaf nodes at hop-1 and hop-2.

Feature Vector Dimensions of Varying Hop/Region Combinations. The dimensions of feature vectors of varying hop/region combinations are summarized in Table 2. As discussed earlier, hop-1 has 4 spatial regions, hop-2 has three spatial regions and all nodes of hop- 3 form one feature vector. Thus, there are eight hop/region combinations in total. Since there are spatial correlations in regions given in Fig. 3, we apply PCA to regional responses collected from all channels and keep leading components for dimension reduction. We keep 15 components for the LFW dataset and 20 components for the CMU Multi-PIE datasets, respectively. Then, the dimension of each feature vector at hop- 1 and hop-2 is the product of 15 (or 20) and the sum of intermediate and leaf nodes at the associated hop for the LFW (or CMU Multi-PIE) dataset.

\begin{tabular}{|c|c|c|c|c|c|}
\hline Hop/Region & LFW & MPIE & Hop/Region & LFW & MPIE \\
\hline \hline Hop-1 (left eye) & 270 & 360 & Hop-2 (upper stripe) & 1,830 & 2,340 \\
\hline Hop-1 (right eye) & 270 & 360 & Hop-2 (lower stripe) & 1,830 & 2,340 \\
\hline Hop-1 (nose) & 270 & 360 & Hop-2 (vertical strip) & 1,830 & 2,340 \\
\hline Hop-1 (mouth) & 270 & 360 & Hop-3 & 233 & 186 \\
\hline
\end{tabular}

Table 2. Feature vector dimensions for LFW and CMU Multi-PIE.

Performance and Model Size Comparison for LFW. We randomly partition male and original plus augmented female images in the LFW dataset into $80 \%$ (for training) and $20 \%$ (for testing) two sets individually. Then, they are mixed again to form the desired training and testing datasets. This is done to ensure the same gender percentages in training and testing. We train eight individual hop/region LR classifiers and one meta LR classifier for ensembles. Then, we apply them to the test data to find out their performance. We repeat the same process four times to get the mean testing accuracy and the standard deviation value, and report the testing performance of each individual hop/region in Table 3 ,

The mean testing accuracy ranges from $82.90 \%$ (hop-1/nose) to $92.42 \%$ (hop$2 /$ vertical stripe). The standard deviation is relatively small. Furthermore, we see that hop- 2 and hop-3 classifiers perform better than hop-1 classifiers. Based on 


\begin{tabular}{|c|c|c|c|}
\hline Classifier & Accuracy(\%) & Classifier & Accuracy (\%) \\
\hline \hline Hop-1 (left eye) & $86.70 \pm 0.65$ & Hop-2 (upper stripe) & $92.25 \pm 0.22$ \\
\hline Hop-1 (right eye) & $86.14 \pm 0.66$ & Hop-2 (lower stripe) & $89.70 \pm 0.73$ \\
\hline Hop-1 (nose) & $82.90 \pm 0.61$ & Hop-2 (vertical strip) & $92.42 \pm 0.56$ \\
\hline Hop-1 (mouth) & $83.42 \pm 0.74$ & Hop-3 & $91.22 \pm 0.46$ \\
\hline
\end{tabular}

Table 3. Performance comparison of each individual hop/region classifier for LFW.

this observation, we consider two ensemble methods. In the first scheme, called FaceHop I, we fuse soft decisions of all eight hop/region classifiers with a meta classifier. In the second scheme, called FaceHop II, we only fuse soft decisions of four hop/region classifiers from hop- 2 and hop- 3 only. The testing accuracy and the model sizes of LeNet-5, FaceHop I and FaceHop II are compared in Table 4. FaceHop I and FaceHop II outperform LeNet-5 in terms of classification accuracy by $0.70 \%$ and $0.86 \%$, respectively, where their model sizes are only about $33.7 \%$ and $22.2 \%$ of LeNet-5. Clearly, FaceHop II is the favored choice among the three for its highest testing accuracy and smallest model size.

\begin{tabular}{|l|c|c|}
\hline Method & Accuracy (\%) & Model Size \\
\hline \hline LeNet-5 & $93.77 \pm 0.43$ & 75,846 \\
\hline FaceHop I (all three hops) & $94.47 \pm 0.54$ & 25,543 \\
\hline FaceHop II (hop-2 \& hop-3 only) & $94.63 \pm 0.47$ & 16,895 \\
\hline
\end{tabular}

Table 4. Performance comparison of LeNet-5, FaceHop I and FaceHop II in accuracy rates and model sizes for LFW.

Performance and Model Size Comparison for CMU Multi-PIE. Next, we show the classification accuracy of each individual hop/region classifier for the CMU Multi-PIE dataset in Table 5. Their accuracy values range from $63.02 \%$ (hop-1/mouth) to $91.95 \%$ (hop-2/upper stripe). It appears that CMU Multi-PIE is more challenging than LFW if we focus on the performance of each individual classifier by comparing Tables 3 and 5 .

\begin{tabular}{|c|c|c|c|}
\hline Classifier & Accuracy (\%) & Classifier & Accuracy (\%) \\
\hline \hline Hop-1 (left eye) & $79.33 \pm 0.33$ & Hop-2 (upper stripe) & $91.95 \pm 0.18$ \\
\hline Hop-1 (right eye) & $78.64 \pm 0.25$ & Hop-2 (lower stripe) & $87.00 \pm 0.15$ \\
\hline Hop-1 (nose) & $65.19 \pm 0.36$ & Hop-2 (vertical strip) & $91.34 \pm 0.22$ \\
\hline Hop-1 (mouth) & $63.02 \pm 0.41$ & Hop-3 & $84.55 \pm 0.77$ \\
\hline
\end{tabular}

Table 5. Performance comparison of each individual hop/region classifier for CMU Multi-PIE. 
We consider two ensemble schemes as done before. FaceHop I uses all eight soft decisions while FaceHop II takes only four soft decisions from hop-2 and hop-3. The mean accuracy performance of LeNet-5, FaceHop I and FaceHop II are compared in Table 6. It is interesting to see that FaceHop I and II have slightly better ensemble results of CMU Multi-PIE than of LFW, respectively. The performance of LeNet-5 also increases from $93.77 \%$ (LFW) to $95.08 \%$ (CMU Multi-PIE). As far as the model size is concerned, the model sizes of FaceHop I and FaceHop II are about $38.4 \%$ and $23.2 \%$ of LeNet-5, respectively. Again, FaceHop II is the most favored solution among the three for its highest testing accuracy and smallest model size.

\begin{tabular}{|l|c|c|}
\hline Method & Accuracy (\%) & Model Size \\
\hline \hline LeNet-5 & 95.08 & 75,846 \\
\hline FaceHop I (all three hops) & $95.09 \pm 0.24$ & 29,156 \\
\hline FaceHop II (hop-2 and hop-3 only) & $95.12 \pm 0.26$ & 17,628 \\
\hline
\end{tabular}

Table 6. Performance comparison of LeNet-5, FaceHop I and FaceHop II in accuracy rates and model sizes for CMU Multi-PIE.

\section{Conclusion and Future Work}

A light-weight low-resolution face gender classification method, called FaceHop, was proposed. This solution finds applications in resource-constrained environments with limited networking and computing. FaceHop has several desired characteristics, including a small model size, a small training data amount, low training complexity, and low-resolution input images. The effectiveness of the FaceHop method for gender classification was demonstrated by experiments on two benchmarking datasets.

In this paper, we demonstrated the potential of the SSL principle for effective feature extraction from face images. As to future work, we would like to test more datasets for gender classification and also extend the SSL principle for identifying heterogeneous and correlated face attributes such as gender, age, and race. It is particularly interesting to develop a multi-task learning approach. Furthermore, it will be desired to work on high-resolution face images and see whether we can get significant performance improvement using the SSL principle in classification accuracy, computational complexity, and memory usage.

\section{References}

1. A. K. Jain, S. C. Dass, and K. Nandakumar, "Soft biometric traits for personal recognition systems," in Biometric Authentication, D. Zhang and A. K. Jain, Eds. Berlin, Heidelberg: Springer Berlin Heidelberg, 2004, pp. 731-738. 
2. D. Riccio, G. Tortora, M. De Marsico, and H. Wechsler, "Ega- ethnicity, gender and age, a pre-annotated face database," in 2012 IEEE Workshop on Biometric Measurements and Systems for Security and Medical Applications (BIOMS) Proceedings. IEEE, 2012, pp. 1-8.

3. E. Gonzalez-Sosa, J. Fierrez, R. Vera-Rodriguez, and F. Alonso-Fernandez, "Facial soft biometrics for recognition in the wild: Recent works, annotation, and cots evaluation," IEEE Transactions on Information Forensics and Security, vol. 13, no. 8, pp. 2001-2014, 2018.

4. C.-C. J. Kuo, "Understanding convolutional neural networks with a mathematical model," Journal of Visual Communication and Image Representation, vol. 41, pp. 406-413, 2016.

5. —_ "The cnn as a guided multilayer recos transform [lecture notes]," IEEE signal processing magazine, vol. 34, no. 3, pp. 81-89, 2017.

6. C.-C. J. Kuo, M. Zhang, S. Li, J. Duan, and Y. Chen, "Interpretable convolutional neural networks via feedforward design," Journal of Visual Communication and Image Representation, vol. 60, pp. 346-359, 2019.

7. Y. Chen, M. Rouhsedaghat, S. You, R. Rao, and C.-C. J. Kuo, "Pixelhop++: A small successive-subspace-learning-based (ssl-based) model for image classification," arXiv preprint arXiv:2002.03141, 2020.

8. S. Gutta, H. Wechsler, and P. J. Phillips, "Gender and ethnic classification of face images," in Proceedings Third IEEE International Conference on Automatic Face and Gesture Recognition. IEEE, 1998, pp. 194-199.

9. B. Moghaddam and M.-H. Yang, "Learning gender with support faces," IEEE Transactions on Pattern Analysis and Machine Intelligence, vol. 24, no. 5, pp. 707-711, 2002.

10. S. Baluja and H. A. Rowley, "Boosting sex identification performance," International Journal of computer vision, vol. 71, no. 1, pp. 111-119, 2007.

11. V. Štruc and N. Pavešić, "Gabor-based kernel partial-least-squares discrimination features for face recognition," Informatica, vol. 20, no. 1, pp. 115-138, 2009.

12. T. Jabid, M. H. Kabir, and O. Chae, "Gender classification using local directional pattern (ldp)," in 2010 20th International Conference on Pattern Recognition. IEEE, 2010, pp. 2162-2165.

13. J. Wu, W. A. Smith, and E. R. Hancock, "Facial gender classification using shapefrom-shading," Image and Vision Computing, vol. 28, no. 6, pp. 1039-1048, 2010.

14. D. Cao, R. He, M. Zhang, Z. Sun, and T. Tan, "Real-world gender recognition using multi-order lbp and localized multi-boost learning," in IEEE International Conference on Identity, Security and Behavior Analysis. IEEE, 2015, pp. 1-6.

15. Y. Li, R. Wang, H. Liu, H. Jiang, S. Shan, and X. Chen, "Two birds, one stone: Jointly learning binary code for large-scale face image retrieval and attributes prediction," in Proceedings of the IEEE International Conference on Computer Vision, 2015, pp. 3819-3827.

16. S. Jia and N. Cristianini, "Learning to classify gender from four million images," Pattern recognition letters, vol. 58, pp. 35-41, 2015.

17. M. Castrillón-Santana, J. Lorenzo-Navarro, and E. Ramón-Balmaseda, "Descriptors and regions of interest fusion for in-and cross-database gender classification in the wild," Image and Vision Computing, vol. 57, pp. 15-24, 2017.

18. B. Lee, S. Z. Gilani, G. M. Hassan, and A. Mian, "Facial gender classification - analysis using convolutional neural networks," in 2019 Digital Image Computing: Techniques and Applications (DICTA). IEEE, 2019, pp. 1-8. 
19. G. Levi and T. Hassner, "Age and gender classification using convolutional neural networks," in Proceedings of the IEEE conference on computer vision and pattern recognition workshops, 2015, pp. 34-42.

20. M. Duan, K. Li, C. Yang, and K. Li, "A hybrid deep learning cnn-elm for age and gender classification," Neurocomputing, vol. 275, pp. 448-461, 2018.

21. F. Taherkhani, N. M. Nasrabadi, and J. Dawson, "A deep face identification network enhanced by facial attributes prediction," in The IEEE Conference on Computer Vision and Pattern Recognition (CVPR) Workshops, June 2018.

22. H. Han, A. K. Jain, F. Wang, S. Shan, and X. Chen, "Heterogeneous face attribute estimation: A deep multi-task learning approach," IEEE transactions on pattern analysis and machine intelligence, vol. 40, no. 11, pp. 2597-2609, 2017.

23. R. Ranjan, V. M. Patel, and R. Chellappa, "Hyperface: A deep multi-task learning framework for face detection, landmark localization, pose estimation, and gender recognition," IEEE Transactions on Pattern Analysis and Machine Intelligence, vol. 41, no. 1, pp. 121-135, 2017.

24. G. Antipov, M. Baccouche, S.-A. Berrani, and J.-L. Dugelay, "Effective training of convolutional neural networks for face-based gender and age prediction," Pattern Recognition, vol. 72, pp. 15-26, 2017.

25. J. Cheng, Y. Li, J. Wang, L. Yu, and S. Wang, "Exploiting effective facial patches for robust gender recognition," Tsinghua Science and Technology, vol. 24, no. 3, pp. 333-345, 2019.

26. F. N. Iandola, S. Han, M. W. Moskewicz, K. Ashraf, W. J. Dally, and K. Keutzer, "Squeezenet: Alexnet-level accuracy with 50x fewer parameters andi 0.5 mb model size," arXiv preprint arXiv:1602.07360, 2016.

27. A. Krizhevsky, I. Sutskever, and G. E. Hinton, "Imagenet classification with deep convolutional neural networks," in Advances in neural information processing systems, 2012, pp. 1097-1105.

28. X. Wu, R. He, Z. Sun, and T. Tan, "A light CNN for deep face representation with noisy labels," IEEE Transactions on Information Forensics and Security, vol. 13, no. 11, pp. 2884-2896, 2018.

29. M. Turk and A. Pentland, "Face recognition using eigenfaces," in Proceedings. 1991 IEEE computer society conference on computer vision and pattern recognition, 1991, pp. 586-587.

30. T.-H. Chan, K. Jia, S. Gao, J. Lu, Z. Zeng, and Y. Ma, "Pcanet: A simple deep learning baseline for image classification?" IEEE transactions on image processing, vol. 24, no. 12, pp. 5017-5032, 2015.

31. Y. Chen and C.-C. J. Kuo, "Pixelhop: A successive subspace learning (ssl) method for object recognition," Journal of Visual Communication and Image Representation, p. 102749, 2020.

32. C.-C. J. Kuo and Y. Chen, "On data-driven Saak transform," Journal of Visual Communication and Image Representation, vol. 50, pp. 237-246, 2018.

33. D. E. King, "Dlib-ml: A machine learning toolkit," Journal of Machine Learning Research, vol. 10, pp. 1755-1758, 2009.

34. G. B. Huang, M. Ramesh, T. Berg, and E. Learned-Miller, "Labeled faces in the wild: A database for studying face recognition in unconstrained environments," University of Massachusetts, Amherst, Tech. Rep. 07-49, October 2007.

35. C. Ferrari, G. Lisanti, S. Berretti, and A. Del Bimbo, "Effective 3d based frontalization for unconstrained face recognition," in 2016 23rd International Conference on Pattern Recognition (ICPR). IEEE, 2016, pp. 1047-1052.

36. R. Gross, I. Matthews, J. Cohn, T. Kanade, and S. Baker, "Multi-pie," Image and Vision Computing, vol. 28, no. 5, pp. 807-813, 2010. 